\title{
HIDROCOLPO POR ECTOPIA URETERAL EM HEMIVAGINA ASSOCIADO A DISPLASIA RENAL: RELATO DE CASO E REVISÃO DA LITERATURA*
}

\author{
Mario Flores Barba ${ }^{1}$, Renata Emy Ogawa ${ }^{2}$, Uenis Tanuri ${ }^{3}$, Anna Tanuri ${ }^{4}$, Vera H. Koch ${ }^{5}$, \\ Luiz Antônio Nunes de Oliveira ${ }^{6}$
}

\begin{abstract}
Resumo Os autores apresentam um caso de displasia renal associada a ectopia ureteral vaginal homolateral, com uretero-hidronefrose contralateral devida a compressão por hidrocolpo causado pela ectopia e por um septo vaginal oblíquo. Os exames de imagem utilizados foram urografia excretora, cistografia, ultra-sonografia, tomografia computadorizada e cintilografia renal com Tc-99m-DMSA. Foi realizada revisão da literatura sobre casos de hidrocolpo associado a malformações urinárias.

Unitermos: Hidrocolpo; Malformação vaginal; Displasia renal.
\end{abstract}

\begin{abstract}
Congenital hydrocolpos due to ectopic ureter in hemivagina associated with renal dysplasia: a case report and review of the literature.

The authors report a case of congenital hydrocolpos due to vaginal malformation (vaginal septae) associated with ectopic ureter and renal displasia. Several imaging diagnostic procedures (ultrasonography, nuclear medicine, intravenous urography and computed tomography) were performed. A review of the literature on hydrocolpos and its association with urinary malformations is also presented.

Key words: Hydrocolpos; Vaginal malformation; Renal displasia.
\end{abstract}

\section{INTRODUÇÃO}

O hidrocolpo congênito é entidade rara que consiste de dilatação cística da vagina devida a obstrução desta, mais freqüentemente causada por atresia vaginal ou hímen imperfurado, e acúmulo de secreção mucóide. Mais raramente, a obstrução é causada por septação vaginal, cisto de Gartner, etc. A presença de hormônios maternos no sangue do recém-nascido é responsável pela intensa secreção de muco pelas glândulas cervicais e vaginais acima

* Trabalho realizado no Serviço de Diagnóstico por Imagem do Instituto da Criança do Hospital das Clínicas da Faculdade de Medicina da Universidade de São Paulo (ICR/HC-FMUSP), São Paulo, SP.

1. Doutor em Medicina pela FMUSP, Assistente Doutor do Serviço de Diagnóstico por Imagem do ICR/HC-FMUSP, Membro Titular do Colégio Brasileiro de Radiologia e Diagnóstico por Imagem (CBR).

2. Médica Residente do Instituto de Radiologia (InRad) do HCFMUSP.

3. Professor Doutor pela FMUSP, Assistente do Serviço de Cirurgia Pediátrica do ICR/HC-FMUSP.

4. Médica Assistente do Serviço de Cirurgia Pediátrica do ICR/ HC-FMUSP.

5. Doutora em Medicina pela FMUSP, Chefe da Unidade de Nefrologia Pediátrica do ICR/HC-FMUSP.

6. Chefe do Serviço de Diagnóstico por Imagem do ICR/HCFMUSP, Membro Titular do CBR.

Endereço para correspondência: Dr. Mario Flores Barba. Alameda Lorena, 1470, ap. 24, Jardim Paulista. São Paulo, SP, 01424-001. E-mail: mf-barba@uol.com.br

Recebido para publicação em 19/32003. Aceito, após revisão, em 26/6/2003. da obstrução. O hidrocolpo comumente está associado a malformações de outros órgãos e sistemas, mais freqüentemente dos tratos urogenital e digestivo, coração e membros ${ }^{(1-3)}$.

A ectopia ureteral ocorre quando o ureter nasce em qualquer local que não no trígono vesical. Está associada a duplicidade pielo-ureteral completa em $80 \%$ dos casos e é mais comum no sexo feminino. Entre as ectopias, a ectopia ureteral vaginal simples é uma forma rara e sua associação com hidrocolpo é mais rara ainda, tendo sido publicados somente quatro casos ${ }^{(4)}$.

A hipoplasia renal com displasia é regra em casos de ectopia ureteral no trato genital, à semelhança do que ocorre na unidade superior dos rins com duplicidade pielo-ureteral total cujo ureter drena ectopicamente $^{(\mathbf{5 , 6})}$.

\section{RELATO DO CASO}

Paciente do sexo feminino proveniente da Unidade de Nefrologia Pediátrica do ICR/HC-FMUSP para realizar exames de imagem de controle de hidronefrose.

Ela vinha com o diagnóstico pré-natal de hidronefrose bilateral, sem intervenções durante a gestação. Nasceu de parto vagi- nal, sem complicações no período neonatal. Apresentava, no exame físico ao nascimento, sopro sistólico e fosseta sacral, sem outras alterações. Evoluiu sem queixas urinárias, e no primeiro exame ultra-sonográfico após o nascimento, com cerca de um mês de vida, evidenciou-se discreta dilatação pielo-calicinal à esquerda, associada a megaureter do mesmo lado e rim direito de tamanho reduzido e com cistos corticais. A uretrocistografia miccional, realizada na mesma época, levantava a suspeita de elevação do assoalho vesical.

A partir dos seis meses de idade a mãe observou aumento do intervalo entre as micções.

Para a investigação do caso foram realizados exames por métodos de imagem, na seguinte ordem: a) cintilografia renal com ácido dimercaptossuccínico de tecnécio99m (Tc-99m-DMSA); b) urografia excretora com contraste iodado não-iônico; c) cistografia; d) ultra-sonografia das vias urinárias; e) tomografia computadorizada com contraste endovenoso iodado não-iônico do abdome e pelve.

A cintilografia renal com Tc-99mDMSA mostrava que o rim direito era responsável por apenas $2 \%$ da sua função, e o esquerdo, por $98 \%$ (Figura 1). 


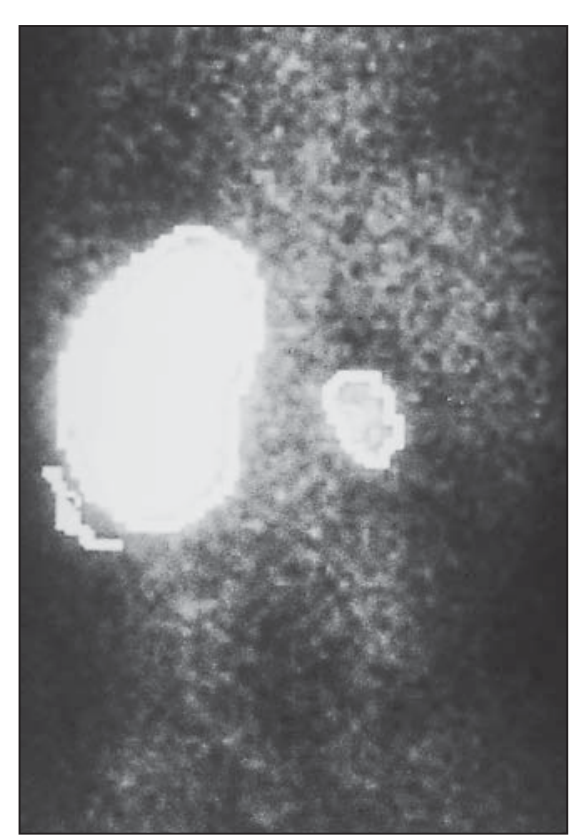

Figura 1. Cintilografia renal com Tc-99m-DMSA. Imagem posterior. Rim direito de tamanho e função reduzidos.

$\mathrm{Na}$ urografia excretora observavam-se exclusão funcional renal à direita, moderada uretero-hidronefrose do rim esquerdo, ureter distal esquerdo com sinais de compressão por massa pélvica radiotransparente, e bexiga desviada e comprimida para a direita e com o seu assoalho elevado (Figura 2).

A cistografia mostrava compressão e desvio da bexiga para a direita e para a frente (Figura 3).

A ultra-sonografia abdominal evidenciava rim direito de tamanho reduzido $(3,5$ $\times 1,6 \mathrm{~cm}$ ) e com alguns cistos corticais, rim esquerdo hidronefrótico e com megaureter, bexiga comprimida por massa cística, de conteúdo homogêneo anecóide, de contornos regulares, que ocupava a pequena pelve (Figuras 4, 5 e 6).

A tomografia computadorizada de abdome e pelve demonstrava massa hipoatenuante pélvica de aproximadamente $10 \mathrm{~cm}$ no seu maior eixo, estando localizada entre a bexiga anteriormente e o reto posteriormente, sem sinais de invasão destes (Figuras 7 e 8).

A paciente foi submetida a laparotomia exploradora e os achados intra-operatórios foram de massa cística de $10 \mathrm{~cm}$, posteriormente à bexiga e anteriormente ao reto, co-

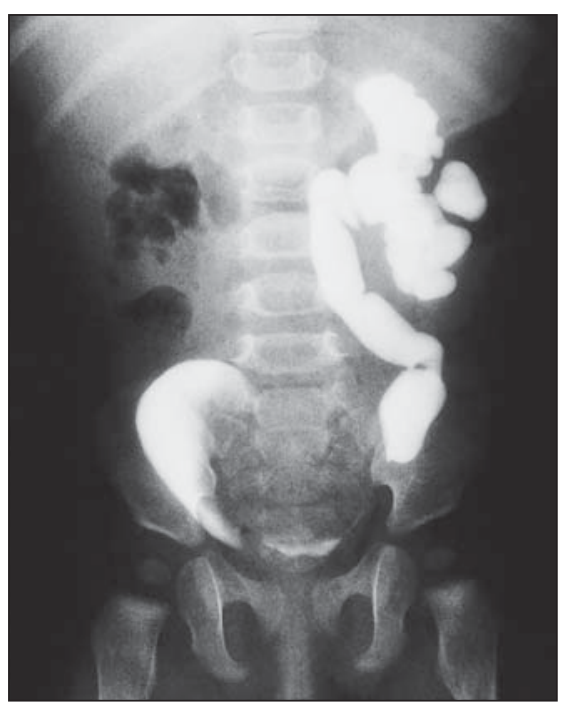

Figura 2. Urografia excretora. Imagem obtida após 60 minutos da injeção endovenosa do meio de contraste. A imagem renal à direita não foi obtida mesmo em radiografias realizadas mais tardiamente (até 360 minutos). Observa-se dilatação acentuada do sistema pielo-calicinal-ureteral à esquerda. Com a bexiga pouco repleta, pode-se observar compressão de sua parede esquerda e desvio para a direita.

municando-se com o útero superiormente. A massa foi puncionada, retirando-se líquido amarelo. Realizando-se toque combinado (o dedo superior entrando pela cavidade do cisto e outro pela vagina), fezse o diagnóstico de duplicação vaginal com intróito vaginal único, sendo que a porção esquerda terminava superiormente em fundo cego e a porção direita correspondia à massa cística (Figura 9). O ureter direito desembocava na hemivagina direita e o rim correspondente era pequeno. A conduta cirúrgica foi ressecção do septo vaginal por via endovaginal e nefroureterectomia direita.

Os diagnósticos pós-operatórios foram urocolpo por duplicidade vaginal (esta causada por septo vaginal oblíquo) e drenagem anômala do ureter direito na hemivagina correspondente, rim direito de tamanho reduzido e uretero-hidronefrose à esquerda.

O diagnóstico anatomopatológico da peça cirúrgica foi de rim displásico tipo II na classificação de Potter.

\section{DISCUSSÃO}

A apresentação clínica do hidrocolpo é de massa palpável em hipogástrio e, em casos de hímen imperfurado, massa vaginal, podendo ser já evidentes as suas complicações, que são compressão de bexiga, ureteres, alças intestinais, vasos sanguíneos pélvicos, diafragma, causando retenção urinária, constipação, edema de membros inferiores e desconforto respiratório ${ }^{(\mathbf{1}, 3)}$. Outras causas de massa vaginal incluem prolapso de uretra, cisto parauretral, prolapso de ureterocele, cisto de Gartner e rabdomiossarcoma de vagina ${ }^{(3)}$.

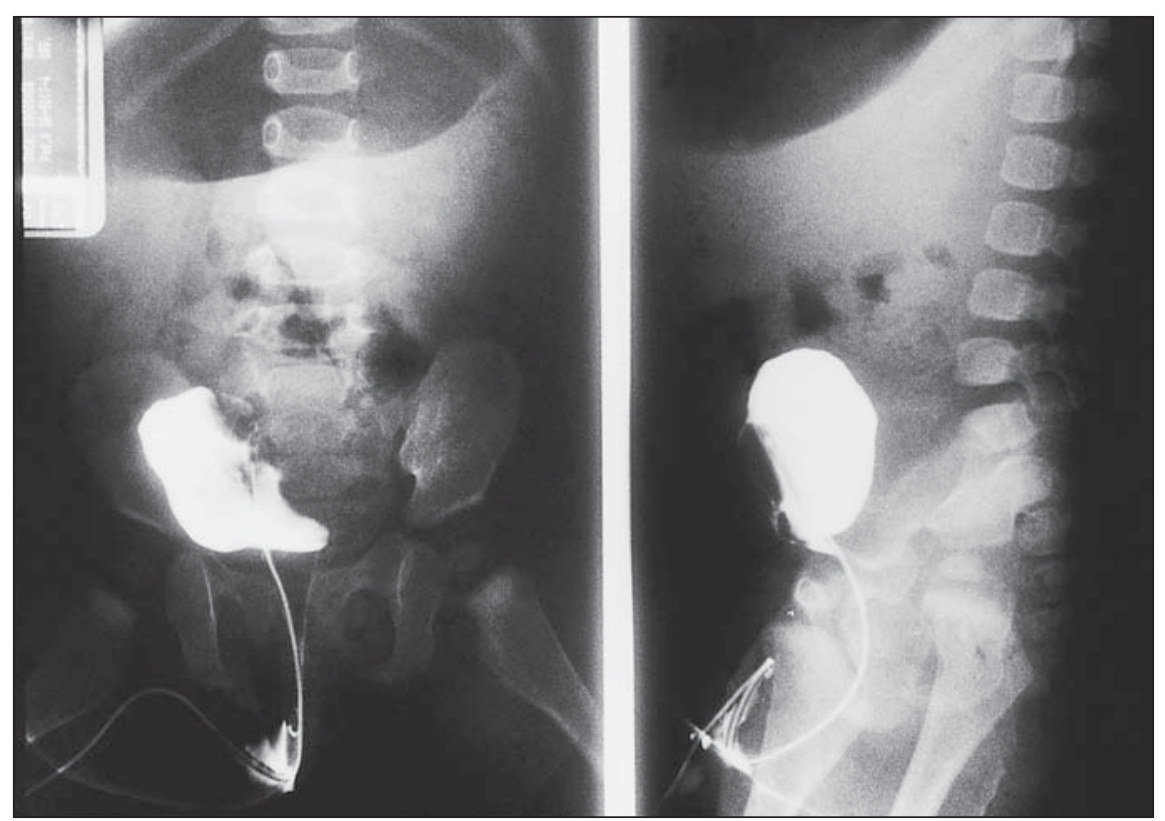

Figura 3. Cistografia. A bexiga cheia deslocada para a direita e anteriormente. 


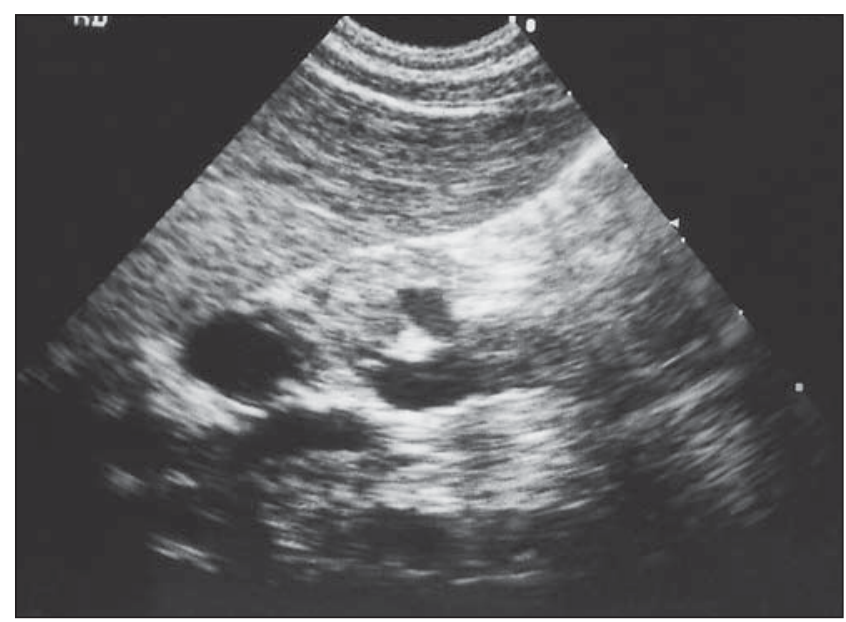

Figura 4. Ultra-sonografia. Imagem longitudinal do rim direito de tamanho reduzido e com cistos corticais simples.

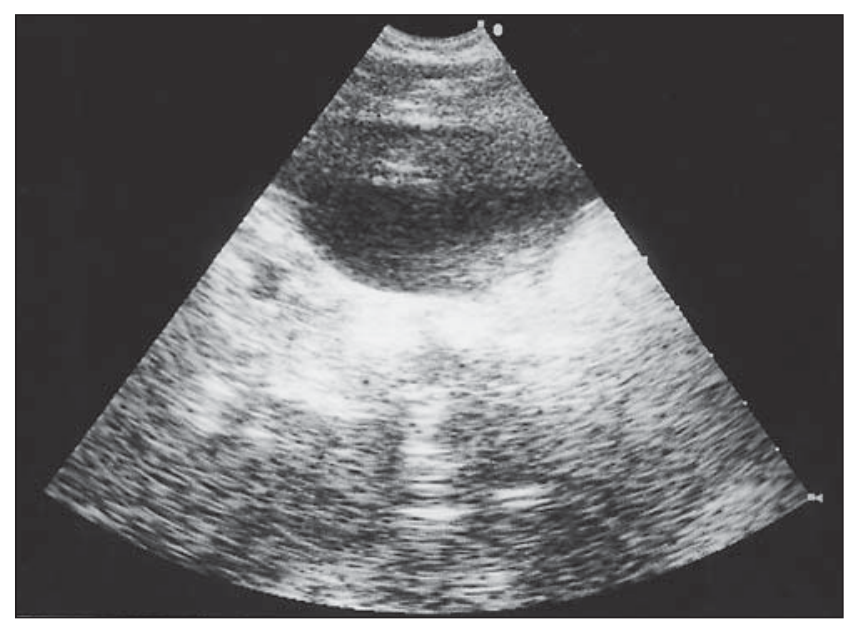

Figura 6. Ultra-sonografia pélvica. Imagem transversa da massa cística, que apresenta paredes finas e conteúdo anecóide. A bexiga não aparece nessa imagem.

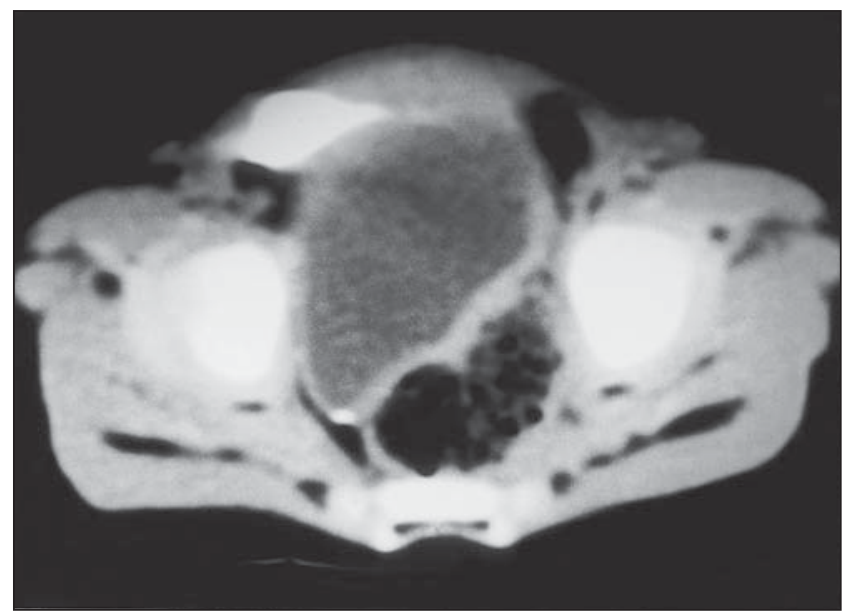

Figura 8. Tomografia computadorizada. À direita ainda se observa a bexiga comprimida, e na região mediana, a massa continuando-se em direção ao períneo.

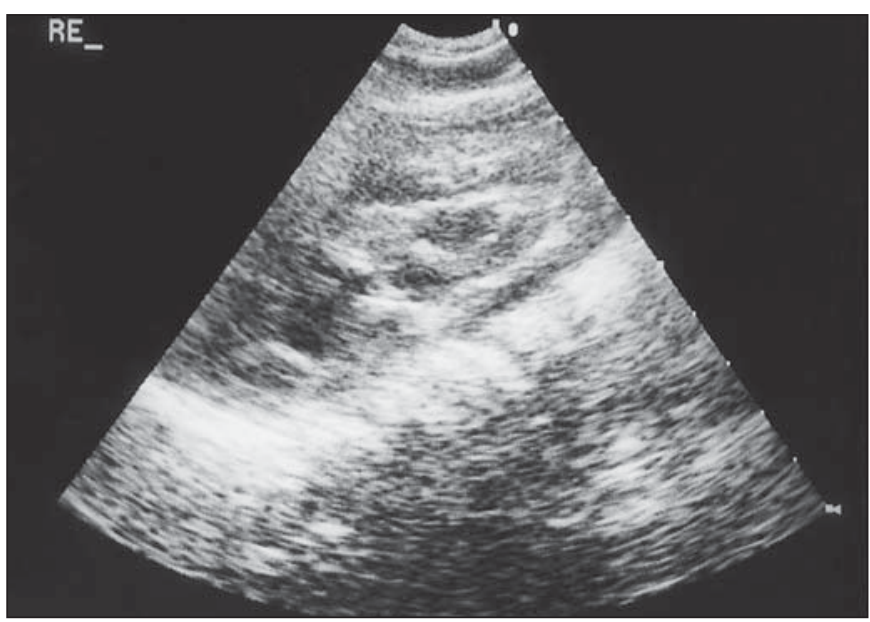

Figura 5. Ultra-sonografia. Imagem longitudinal do rim esquerdo com sinais de hidronefrose.

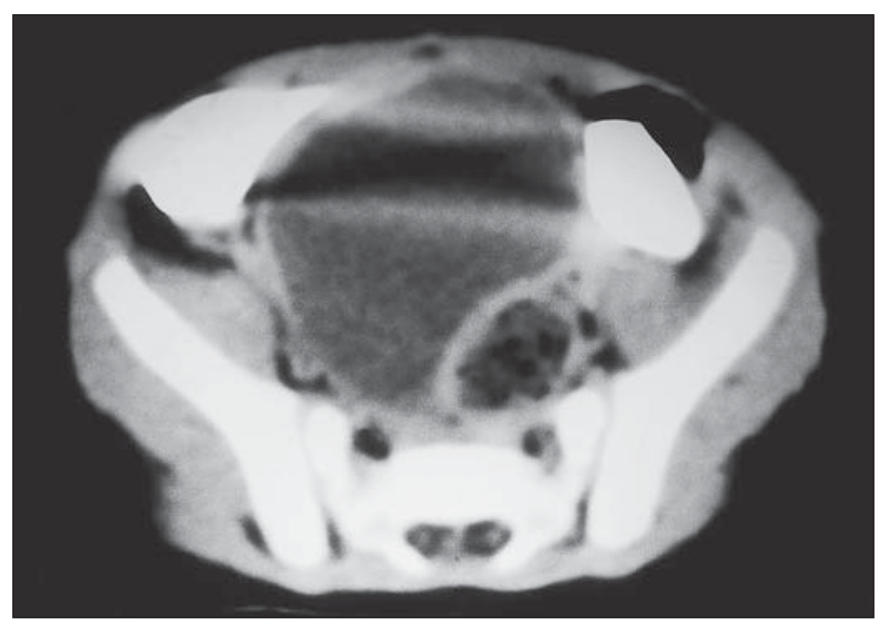

Figura 7. Tomografia computadorizada. Observa-se contrastação da bexiga urinária, que se encontra rechaçada para a direita e do ureter esquerdo, que se encontra dilatado. Notar a imagem de massa arredondada, de paredes finas, conteúdo homogêneo e hipoatenuante, que não se realça com a injeção do contraste. Ela está situada posteriormente à bexiga e anteriormente ao reto.

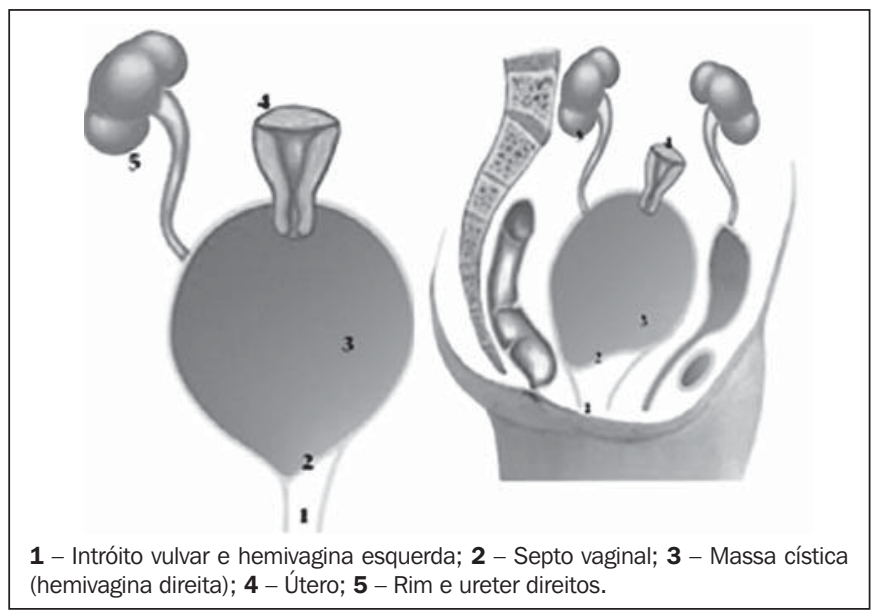

Figura 9. llustração do achado intra-operatório. 0 rim esquerdo está mostrado anteriormente, apenas para melhor visualização. 0 rim direito drena ectopicamente para a hemivagina direita e o rim esquerdo drena normalmente para a bexiga. 
O diagnóstico precoce, neste caso, foi dificultado pelo fato de o hidrocolpo ser muito pequeno ao nascimento, não causando sintomas ou alterações expressivas de exames físico e de imagem (apenas suspeita de elevação do assoalho vesical na uretrocistografia miccional). Somente após o hidrocolpo "ter-se tornado" um urocolpo (acúmulo de urina na vagina) e começado a comprimir o ureter do rim funcionante e a dar sintomas é que o diagnóstico foi possível. Apesar disso, o diagnóstico de certeza não foi possível previamente à cirurgia, já que os órgãos genitais internos do lactente em questão não eram acessíveis pelos métodos de imagem utilizados. Mesmo o vaginograma não acrescentaria muitos dados a esse caso, e possivelmente deixaria a hipótese diagnóstica de hidrocolpo em segundo plano, já que as causas mais comuns de obstrução - atresia vaginal e hímen imperfurado - seriam afastadas pela demonstração de uma (hemi)vagina.

A imagem ultra-sonográfica do hidrocolpo é muito semelhante à da bexiga cheia, e se ela não estiver cheia durante o exame, o hidrocolpo passa despercebido.

Atualmente, para investigação de hidronefrose e massas císticas na pelve, além da ultra-sonografia, vem sendo indicada a urorressonância magnética com seqüências rápidas HASTE ("half Fourier acquisition single-shot turbo spin-echo") e SS-FSE ("single- shot fast spin-echo"), por se tratar de estruturas cheias de líquido com muito baixo fluxo ${ }^{(7)}$. Pode-se fazer avaliação anatômica e funcional das vias urinárias com o uso de contraste endovenoso.
A explicação embriológica para este caso é complexa. O desenvolvimento normal dos ductos paramesonéfricos (ou ductos de Müller, que vão originar alguns dos órgãos genitais femininos internos) só ocorre após o desenvolvimento normal dos ductos mesonéfricos (ou ductos de Wolff, que vão originar alguns dos órgãos genitais internos masculinos e as vias urinárias nos dois sexos). Para haver simultaneamente duplicação vaginal e agenesia/hipoplasia renal, o estímulo teratogênico deve ocorrer no início da quarta semana de gestação. O teratógeno leva à diferenciação defeituosa dos ductos de Wolff e dos brotos ureterais, causando interrupções no desenvolvimento do primórdio uterovaginal, traduzido por fusão incompleta, ou desenvolvimento incompleto, ou não desenvolvimento de partes de um ou de ambos os ductos de Müller e canalização incompleta da placa vaginal. A fusão incompleta desses ductos leva à duplicação genital, que pode resultar em útero didelfo, ou bicórneo, ou septado, ou vagina dupla.

A implantação do ureter na vagina ocorre porque o broto ureteral nasce em porção mais cranial do ducto mesonéfrico e não se separa dele para fundir com a bexiga. A implantação ectópica do ureter na mulher pode ocorrer em qualquer ponto do resquício do ducto mesonéfrico, conhecido como canal de Gartner. Conseqüentemente, pode ocorrer o implante ureteral nas tubas, corpo e cérvix do útero e na vagina, por onde passa o dito canal.

O broto ureteral ectópico pode encontrar o blastema metanéfrico (que originará os néfrons) em um dos pólos renais, ao contrário do que ocorre com o broto ureteral tópico, que encontra o blastema na região central do rim. Como as células dos pólos têm menor poder de diferenciação que as da porção central, pode haver formação de um rim displásico ${ }^{(\mathbf{1 , 4 , 5 , 8})}$.

Os métodos de imagem acima descritos são fundamentais no auxílio diagnóstico e na avaliação pré-operatória neste tipo de síndrome, uma vez que se visualizam de maneira apropriada as alterações orgânicas encontradas nesta condição.

\section{REFERÊNCIAS}

1. Tsukahara H, Fujimoto I, Hiraoka M, et al. Congenital hydrocolpos combined with unilateral renal agenesis. Acta Paediatr Jpn 1991;33:729-33.

2. Schaffer RM, Taylor C, Haller JO, Friedman AP, Shih YH. Nonobstructive hydrocolpos: sonographic appearance and differential diagnosis. Radiology 1983;149:273-8.

3. Bogen DL, Gehris RP, Bellinger MF. Special feature: picture of the month. Denouement and discussion: imperforate hymen with hydrocolpos. Arch Pediatr Adolesc Med 2000;154:959-60.

4. Jaureguizar E, Pereira PL, Santamaria P, Valdes R. Ectopia ureteral vaginal asociada a hidrocolpos y displasia renal. Arch Esp Urol 1987;40:501-4.

5. Bondonny JM, Boissinot F, Vergnes P, Diard F, Sandler B. Association, chez la fille, d'un rein dysplasique avec simple à abouchement ectopique vaginal et d'une anomalie genitale homolatérale. Chir Pédiatr 1988;29:273-80.

6. Ahmed S. Cloacal malformation with solitary crossed renal ectopia and single-system vaginal ectopic ureter. Pediatr Surg Int 1999;15:417-8.

7. Hélénon O, Ghouadni M, Khairoune A, Balleyguier C, Correas JM. Syndromes obstructifs du haut appareil urinaire. Encycl Méd Chir. Radiodiagnostic V - Urologie-Gynécologie, 2002;88.

8. Moore KL, Persaud TVN. O sistema urogenital. In: Moore KL, Persaud TVN. Embriologia clínica. 5aㅗ ed. Rio de Janeiro, RJ: Guanabara Koogan, 1993: 249-83. 